\title{
Sensory Effects of Baroreceptor Activation and Perceived Stress Together Predict Long-Term Blood Pressure Elevations
}

\author{
T. Elbert, B. R. Dworkin, H. Rau, P. Pauli, \\ N. Birbaumer, C. Droste, and C. H. M. Brunia
}

Activating the arterial baroreceptors in animals has been shown to blunt pain sensation and provide other forms of central nervous system inhibition. This study tested the hypothesis that, among human subjects, a tonic increase in blood pressure (BP) could be a learned response to environmental stressors among subjects in whom the baroreceptor inhibitory mechanism is active. In a sample of 96 healthy, normotensive men and women, amount of pain-reduction produced by baroreceptor stimulation predicted an increase in resting BP 20 months later; the increase was proportional to self-assessed daily life stress. Among the subjects reporting the greatest amount of stress, the pain inhibition effect accounted for more than $80 \%$ of the BP variance. These results support the hypothesis that the reduction in perceived stress produced by baroreceptor stimulation may reward learned increases in BP.

T. Elbert, University of Münster, Institute of Experimental Audiology, Münster, Germany; B. R. Dworkin, Department of Behavioral Science, Department of Neuroscience, and Department of Psychology, Pennsylvania State University College of Medicine, Hershey, PA, USA; H. Rau and P. Pauli, Eberhard-Karls Universität Tübingen, Institute for Medical Psychology and Behavioral Neuroscience, Tübingen, Germany: N. Birbaumer, Eberhard-Karls Universität Tübingen, Institute for Medical Psychology and Behavioral Neuroscience, Tübingen, Germany, and Università degli Studi, Dipartimento Psicologia Generale, Padova, Italy; C. Droste, Benedikt Kreuz Rehabilitation Center for Cardiovascular Diseases, Bad Krozingen, Germany; C. H. M. Brunia, Tilburg University, Department of Psychology, Section Physiological Psychology, Tilburg, The Netherlands.

This research was supported by Deutsche Forschungsgemeinshaft Grant EL 101/3 to Thomas Elbert and National Institutes of Health Grant RO1 HL40837 to B. R. Dworkin.

We thank Renate Schweizer, Ping Zhuang, and M. Mueller for help in acquiring the data, and Susan Dworkin, Anne Hawkins, Marshall B. Jones, Peter Kaufmann, and Ralph Norgren for advice on the article.

Correspondence concerning this article should be addressed to B. R. Dworkin, Pennsylvania State University College of Medicine, Room C1720. Hershey, PA 17033. 
Key words: baroreceptor inhibition, blood pressure (BP), learning, stress, hypertension

Baroreceptor stimulation dampens central nervous system (CNS) arousal, and, in situations in which blood pressure (BP) is elevated by behavioral excitation or pain, lowering arousal can help to reduce it. As such, CNS dampening has a regulatory function paralleling the negative feedback effects of the sympathetic and vagal baroreflexes; however, unlike the peripheral reflexes (Eckberg \& Sleight, 1992), which engage comparatively discrete efferent depressor mechanisms, the general behavioral and sensory effects of barostimulation (Dworkin et al., 1994) are mediated by the ascending reticular activating system and involve a broad range of inhibitory phenomena. Attenuation of spinal reflexes, pain perception, anxiety, and sham rage, and the induction of syncope or sleep have all been reported as immediate consequences of barostimulation (Dworkin, Filewich, Miller, Craigmyle, \& Pickering, 1979). Certain common characteristics of these effects suggest that, in addition to lowering BP, baroreceptor activation may share key properties with other stimuli that are known to be effective behavioral reinforcers or rewards.

In stressful situations, the prompt relief of pain and/or anxiety by coping behaviors can be a reward that, through learning, strengthens these behaviors. Effective coping behaviors can be harmless; however, in many instances, although providing relief, they eventually cause substantial damage. The habitual abuse of barbiturates or alcohol are common examples of learned behaviors that effectively relieve pain or anxiety but eventually have undesirable health consequences. It has been found that with appropriate rewards, behaviors leading to elevated BP also can be learned.

Human spinal injury patients (Pickering et al., 1977) can be taught to use a vagal mechanism to avoid episodes of orthostatic hypotension by voluntarily raising BP, and there are a number of convincing studies of baboons and monkeys showing that, using shock avoidance and/or food as rewards, they can be taught to raise and maintain elevated BP (Benson, Herd, Morse, \& Kelleher, 1969; Harris, Gilliam, Findley, \& Brady, 1973; Plumlee, 1969; Turkkan \& Harris, 1981). Because increased BP itself stimulates baroreceptors, and barostimulation inhibits pain and anxiety, it logically follows that repeated episodes of BP-mediated baroactivation could reward and strengthen, through trial and error learning, those behaviors that caused the $\mathrm{BP}$ to rise in the first place. It has been suggested that such learning could be involved in initiating and maintaining essential hypertension (Dworkin, 1988, 1991; Dworkin et al., 1979; Miller \& Dworkin, 1977). Under this baroreceptor reward hypothesis, a person possessing a strong baroreceptor pain inhibition effect is predisposed to learn hypertension as a coping response, just as a person with ready access to narcotics might be predisposed use them. However, a strong baroreceptor CNS inhibition trait by itself is not sufficient. Effective learning would also require conjunction in the same 
individual of the trait with chronic exposure to noxious social or environmental stimuli that are aversive or stressful. A similar conjunction is apparently necessary in experimental models of barbiturate addiction: It has been shown that rats will learn to bar-press to gain infusions of barbiturate, but only if the learning situation includes a background level of aversive stimulation, such as occasional random foot shocks (Miller, Davis, \& Lulenski, 1968). Presumably, this is because the barbiturate infusion rewards the bar-pressing behavior by lowering the anxiety created by the foot shock: If there is no anxiety, then there is no substrate for the anxiolytic action of the barbiturate, and there is no reward for the bar-pressing behavior. Similarly, it would be expected that a BP-elevating behavior, which produces barostimulation, would be rewarded only if there were also a preexistent level of pain and/or anxiety that the barostimulation could ameliorate. Thus, under the baroreceptor reward hypothesis, baroreceptor activation is the reward mechanism, but the presence of aversiveness or stress in the environment actually provides the necessary motivation, and both are required for any learning of hypertension. This specificity of effect generates the unique quantitative predictions that are tested in the subsequent analyses.

\section{IS BAROSTIMULATION A SUFFICIENTLY EFFECTIVE REWARD FOR LEARNING BP-ELEVATING BEHAVIORS?}

Although spinal-injured humans and laboratory animals can learn substantial and sustained BP elevations, these examples both involve special circumstances. The patients were strongly motivated and were given accurate BP information in a carefully designed program of training, and the reward in the animal experiments involved complete avoidance or escape from moderately painful electric shocks. Escaping from shock is an unquestionably dramatic behavioral consequence, which probably has a vastly greater reinforcement effect than barostimulation. Notwithstanding, trial and error learning is ubiquitous, and over sufficient time, behaviors with comparatively modest but consistent advantage can and do incrementally accrue considerable learned strength: The acquisition of musical, athletic, or typing skills are good examples of gradual but substantial trial and error learning with innumerable small rewards; and other more medically relevant examples are the antalgic gait or the limp that emerges (frequently without any clear awareness) following a foot injury (Sutherland, 1984), or learned restraint of heart rate during exercise (Talan \& Engel, 1986). Given these precedents, we hypothesized that for certain individuals, if increasing BP consistently ameliorated the discomforts of a stressful daily life (in the same sense that learning to limp ameliorates the pain of an injury), some of those individuals might learn to use BP-elevating behaviors to help reduce or cope with the aversiveness of events that contributed to their life stress; further- 
more, it seemed at least plausible that with sufficient time, for the more sensitive subjects, the learning might eventually cumulate (Dworkin, 1991) into measurable increases in basal BP.

More specifically, for a particular individual, the baroreceptor reward hypothesis (Dworkin, 1988) predicts a dependence of the magnitude of the BP rise on an interaction between the strength of the baroreceptor CNS inhibition and the experienced level of chronic aversiveness. This means (a) that without any aversiveness, even a large baroreceptor inhibition effect should be without detectable consequence; and (b) without an inhibition effect, aversiveness itself should not influence BP. Quantitatively, the reward and, thus, the BP learning effect are expected to increase roughly in proportion to the product of the two factors. These explicit predictions were tested for 96 subjects using measures of barostimulation pain inhibition (Dworkin et al., 1994), stress, and the change in BP during a 20 -month period.

\section{METHODS}

\section{Subjects}

Normal adults were recruited by newspaper announcements in the Tübingen area and paid $100 \mathrm{DM}$ for participation in the experiment. Exclusion criteria were any kind of chronic or acute disease, including hypertension. This procedure resulted in a sample of 73 men and 47 women: Weight $=69.7 \pm 8.8$ $\mathrm{kg}$; Height $=173.0 \pm 8.8 \mathrm{~cm} ;$ Age $=31.4 \pm 6.0$ years; Mean blood pressure $=$ $103.5 \pm 9.5 \mathrm{mmHg}$. Informed consent was obtained, and all procedures conformed to the human subject regulations of Tübingen University.

\section{Measurement of Pain Attenuation}

The degree to which pain perception was attenuated by baroreceptor activation was estimated for each subject using an external neck chamber in which the pressure was varied in relation to the cardiac cycle. During systole, suction of approximately $-30 \mathrm{mmHg}$ was applied to the chamber; and during diastole, a positive pressure of approximately $10 \mathrm{mmHg}$ was applied. The initial pressure pulse commenced $100 \mathrm{msec}$ after the ventricular R-wave; the systolic pulse was immediately followed by an opposite pulse during diastole: Both pulses were equal in duration to one half of the mean cardiac interbeat interval less $100 \mathrm{msec}$. Following the diastolic pulse, the chamber was vented to atmospheric pressure. This procedure effectively enhances the carotid pulse and decreases the heart rate by approximately $5 \%$. For each of 326 -sec trials during which the alternating pressures were applied on each heart beat, an electrical stimulus 
composed of an optically isolated bipolar 10 -msec pulse was applied to a 1 -mm gold finger-tip electrode at a strength of $120 \%$ of the preestablished pain threshold $(<1 \mathrm{~mA})$. The stimuli were delivered following the second and fourth R-wave of each trial. In half of the trials the stimulus was presented during systole, and in the other half the stimulus was presented during diastole. The two kinds of trials alternated pseudorandomly. At the end of each trial, the subjects were prompted to perform a computerized subjective magnitude estimation of the two stimuli. The vertical visual analog scale ranged from 0 (representing no perception) to 24 (representing substantial pain). The entire procedure required approximately $1 \mathrm{hr}$. The estimate of the individual baroreceptor inhibition effect (baro $\triangle$ PAIN) was defined as the difference between the pain responses to stimuli presented in the systolic and diastolic trials. (The procedures and measurements are identical to those reported in Dworkin et al., 1994; see Figure 1D for the detailed method and statistics of the group data.)

\section{Daily Estimates of Perceived Stress}

Individual levels of perceived aversiveness (STRESS) were based on the median of a series of seven daily at-home estimates recorded at a quiet time each evening by the subjects on a paper form with an integer scale of 0 to 10. The specific question asked was, "Wie stark waren Sie heute belastet $(0=$ wenig $10=$ stark $)$," which approximately translates to "How loaded down did you feel today? $(0=$ little $10=$ very much)." In the American idiom it has the same meaning as "How stressful was your day?" The term stress or "Starke Belastung" had no intended theoretical connotation. It was only a convenient figure of speech to help the subjects identify and estimate the degree of aversiveness that they perceived to characterize their days.

\section{BP Measurements}

For determination of the initial mean arterial pressure (MAP), subjects measured and recorded their own BP with an automated monitoring device; this was done for 7 consecutive days each day immediately following the recording of the STRESS estimate. It was deemed particularly important for this study that the BP estimates be as free as possible of situational stress. Ambulatory BP recording has revealed the large situational variability of BP and has shown that psychosocial factors are an important source of that variability: Measures taken in arousing circumstances, such as in a medical office or laboratory, are known to be poorly correlated with resting BP (Pickering, 1991). In particular, there is known to be a large overestimation of BP from measurements during and after laboratory procedures (Obrist, 
Light, James, \& Strogatz, 1987). Any laboratory BP measurement is thus confounded with a stress response to the situation. Although such a provoked measurement might be useful for diagnoses of hypertension, it was undesirable for this experiment, in which independent estimate of these variables was needed. Teaching the subjects to use automated BP recorders, and having them use them at home at a quiet time each evening, provided the most appropriate estimates of resting pressure. We used a calculated MAP based on the conventional formula: $2 / 3$ diastolic $+1 / 3$ systolic. The use of MAP rather than separate diastolic or systolic pressures slightly improved the stability of the BP data; however, had we instead used either systolic or diastolic separately, the statistical results and conclusions presented here would have been essentially unchanged.

\section{Schedule of Measurements and Computation of the BP Change Score}

The laboratory measurements (baro $\triangle$ PAIN) were completed between February, 1990, and February, 1991. For each subject, the stress estimates (STRESS) and initial BP measurements (MAP) were completed in the 7 days immediately following the laboratory barostimulation session. Approximately 18 months after the laboratory sessions, 107 of the 116 subjects were successfully contacted; and of these, 100 agreed to repeat the seven daily BP readings with the same instrument that they had used for the initial measurement. The difference between the median of these seven readings and the median of the initial seven readings measured the individual's change in BP ( $\triangle$ MAP) during the ensuing $19.7 \pm 3.8$ months.

An alternate procedure for computing the change scores uses the difference between the observed initial MAP and the regression predicted final MAP, rather than between the initial and final raw MAPs. The regression procedure in principle eliminates any contribution to the covariance by the initial score (Cronbach \& Furedy, 1970), but its applicability depends upon homogeneity of the regression across the relevant variables. Because of the presence of a substantial interaction (discussed later), the data did not satisfy that requirement, and we chose to use the simpler procedure; nevertheless, all of the calculations have been done with both the regression and raw score procedures, and the results and conclusions presented later are essentially the same with either method. For reference, Figure 3 gives several key correlations using both baseline-free regression-generated and raw difference score estimates of $\triangle \mathrm{MAP}$.

The data used in the following analyses consist of the 96 completed sets of measurements composed of a laboratory baroreceptor pain inhibition measurement (baro $\triangle \mathrm{PAIN}$ ), a median aversiveness estimate (STRESS), and the change in median MAP ( $\triangle \mathrm{MAP})$. None of the 96 subjects who completed all of the measurements were excluded from any of the analyses. 


\section{RESULTS}

For the 96 subjects, there was a reliable positive linear relation $(r=0.29$, $F=8.8 ; p<.004$ ) between the pain inhibition score (baro $\Delta$ PAIN) and the change in BP $(\triangle \mathrm{MAP})$. Although baro $\triangle \mathrm{PAIN}$ accounted for only $7.5 \%$ of the $\triangle \mathrm{MAP}$ variance (reduced to adjust for the degrees of freedom of the model; Neter, Wasserman, \& Kutner, 1985), the inherent noisiness of both the $\triangle \mathrm{MAP}$ and baro $\triangle \mathrm{PAIN}$ measures and the multitude of other factors that could have influenced an individual's BP during 20 months unquestionably attenuated the relation. For example, the correlation between the initial and final MAP is only .76; using this value to estimate conservatively the $\triangle \mathrm{MAP}$ reliability and correct for attenuation in the usual manner, the actual accounted-for variance would be $\cong 30 \%$ to $45 \%$ larger. We did not use corrections of this kind, and thus the correlations presented are in every instance the most conservative estimates. In any event, the reliable correlation of .29 clearly shows that a component of the pain inhibition measure impacts in some way on long-term BP regulation. But how? One possibility is that baro $\triangle$ PAIN and $\triangle \mathrm{MAP}$ were independently affected by individual baroreflex sensitivity parameters, which were unrelated to CNS inhibition. This, however, is by the usual account not very likely: Although the baroreceptors are most prominently the receptive field of BP stabilizing reflexes, the buffer reflexes themselves, because of resetting, are not thought to participate directly in long-term BP regulation. Furthermore, putting the somewhat controversial (see Eckberg \& Sleight, 1992, pp. 328-331) question of resetting aside, within either a conventional regulatory or a cardiovascular reactivity framework it is especially difficult to explain how higher baroreceptor sensitivity would lead to higher BP, rather than the opposite.

Ultimately, the usefulness of an hypothesis is measured by both its correspondence to the data and the specificity of its predictions (Graham et al,, 1962). Thus, going beyond speculation about other possible artifacts, the prediction of the baroreceptor reward hypothesis is very explicit: If the long-term BP rise in the barosensitive subjects was learned, and if baroreceptor CNS inhibition mediated the reward, then for a given individual the strength of reward would have to have strongly depended on an interaction between the strength of the inhibition (baro $\triangle$ PAIN) and the individual's perceived level of aversiveness or STRESS. This, in fact, is what was found.

The graph in Figure 1 is a plot of a least squares regression surface fit to the baro $\triangle$ PAIN, STRESS, and $\triangle$ MAP data. Following conventional statistical practice, we included both the linear terms and the interaction in the initial model. The resulting surface, which had the form $\triangle \mathrm{MAP}=0.13-$ 0.15 (STRESS $)+0.14$ (baro $\triangle$ PAIN $)+0.26$ (STRESS $\times$ baro $\triangle$ PAIN $)$, ac counted for $7 \%$ of the model adjusted $\triangle$ MAP variance $(r=0.31, F=3.4 ; p<$ $.02)$, which is somewhat less than the variance accounted for by barosensitivity alone. Figure 2, however, shows the almost complete irrele- 


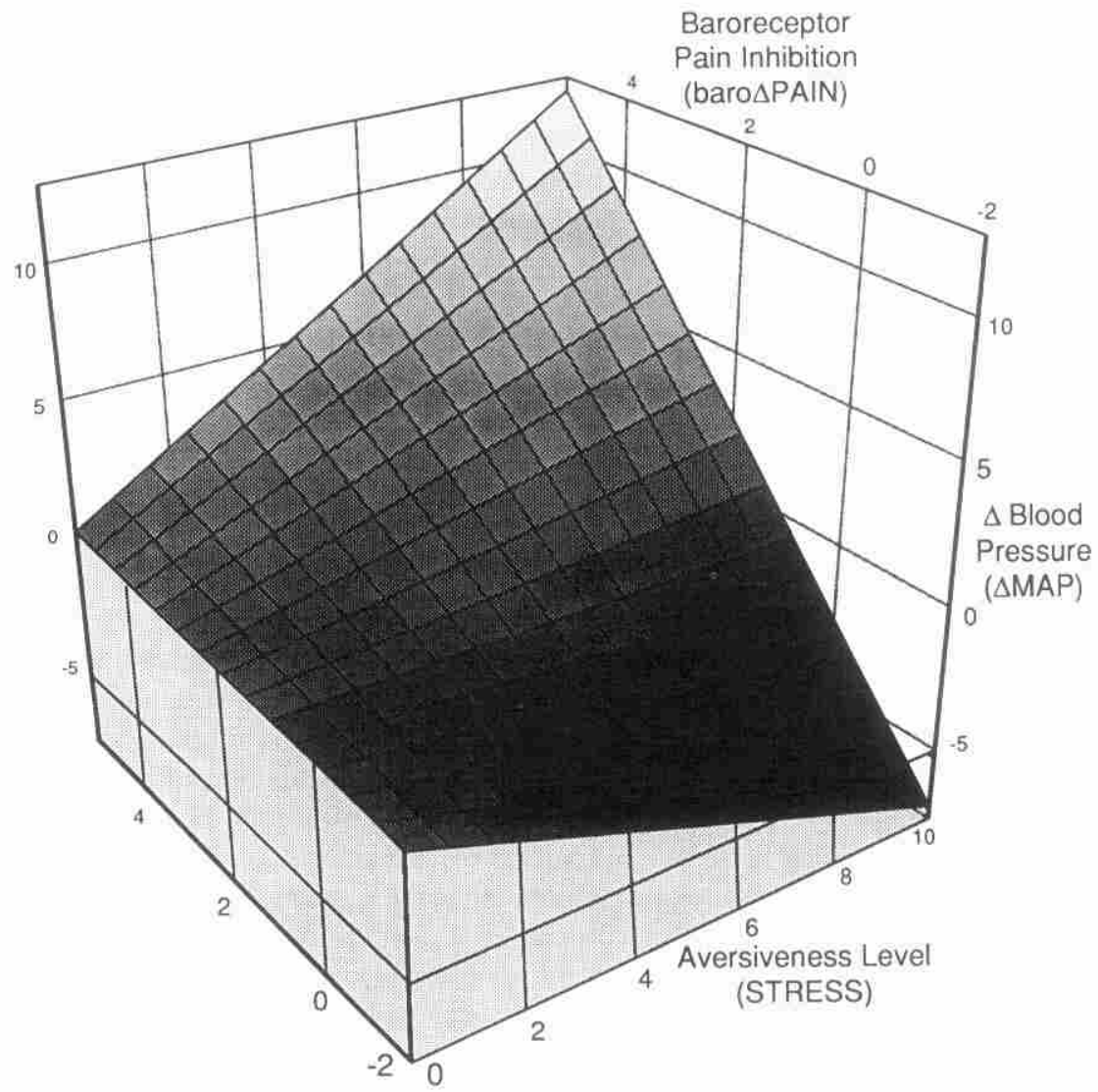

FIGURE 1 Least squares regression surface fit to the Tübingen data. This plot is based on a regression model that included the linear terms, baro $\triangle P A I N$ and STRESS; however, including these terms did not augment the $\triangle \mathrm{MAP}$ variance accounted for by the interaction, baro $\triangle \mathrm{PAIN} \times$ STRESS, alone (see Figure 2 ). This result, based on data from normotensive volunteers, is consistent with the hypothesis that BP-activated, baroreceptor-mediated CNS inhibition can sufficiently reduce the aversiveness of noxious (stressful) stimuli to reward and eventually strengthen BP-elevating behaviors (see text).

vance of the linear terms to the analysis: For example, for those subjects reporting zero STRESS, pain inhibition apparently has virtually no influence on $\triangle \mathrm{MAP}$; and conversely, for subjects with zero baro $\triangle \mathrm{PAIN}$, there is no positive STRESS effect on $\triangle \mathrm{MAP}$. This result implies that the linear terms contributed only to the error variance of the analysis and that a simpler regression model could be more appropriate. In fact, a regression equation containing only the interaction STRESS $\times$ baro $\triangle \mathrm{PAIN}$ and an intercept term conforms exactly to the predictions of the hypothesis, and this model accounts for $8.6 \%$ of the model adjusted $\triangle \mathrm{MAP}$ variance $(r=0.31, F=9.9 ; p$ 
$<.002$ ). (The plot of the least squares regression surface for the pure interaction is not shown but is nearly identical to Figure 1.)

Some of the key features of Figure 1 are possibly more easily appreciated by comparing the baro $\triangle \mathrm{PAIN}-\triangle \mathrm{MAP}$ correlations in selected subgroups of subjects who reported different STRESS levels. The STRESS ratings were on a 0 to 10 integer scale, and the scores were approximately normally distributed; thus an exact median split into two equal groups was not possible. However, arbitrarily splitting the STRESS scale at 5.5 , which is its
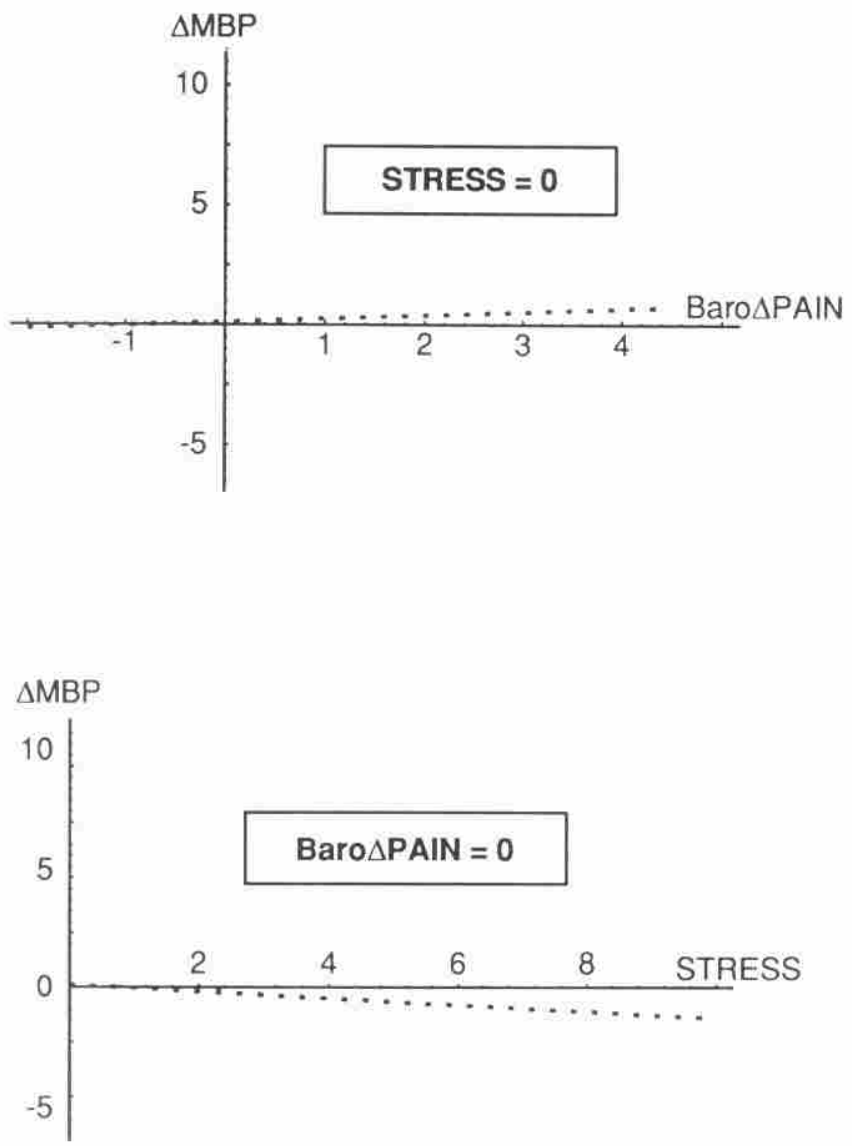

FIGURE 2 The regression of $\triangle M A P$ on baro $\triangle P A I N$ in the plane of STRESS $=0$, and the regression of $\triangle \mathrm{MAP}$ on STRESS in the plane of baro $\triangle \mathrm{PAIN}=0$ for an analysis that includes both linear terms (see Figure 1). The obvious lack of effect in both cases indicates that neither linear term contributes significantly to the variance accounted for by the model. A simpler model, $\triangle \mathrm{MAP}=-0.56+0.26$ (baro $\triangle \mathrm{PAIN} \times \mathrm{STRESS})$, which includes only the interaction, accounts for a larger proportion of the variance when both models are appropriately corrected for the number of variables. 
center, classifies 62 subjects as low stress and 34 as high stress. For the low stress $(<5.5)$ subjects, only $1 \%$ of the variance in $\triangle \mathrm{MAP}$ was accounted for by baro $\triangle$ PAIN ( $r=.11, F=.72 ; p=.40)$; whereas, for the 34 subjects whose stress estimate was above midscale, $29 \%$ of the $\triangle$ MAP variance was accounted for by baro $\triangle$ PAIN $(r=.56, F=14.2 ; p<.001)$. The surface in Figure 1 is especially steep for the highest stress levels, and this coincides well with the actual correlations: For the 17 subjects with STRESS $>7$, approximately $35 \%$ of the adjusted $\triangle$ MAP variance $(r=.63, F=9.7 ; p<.01)$ was accounted for by the baro $\triangle$ PAIN score, and for the eight highest stress subjects (approximately the upper decile), who reported median daily STRESS levels of 9 or 10 , the laboratory baroreceptor pain inhibition measurement (baro $\triangle$ PAIN) accounted for more than $80 \%$ of the adjusted $\triangle$ MAP variance $(r=93, F=$ $15.2 ; p<.01)$. Figure 3 gives additional subgroup correlations, rank order statistics, and correlations with the initial baseline MAP controlled by using the regression calculated $\mathrm{BP}$ over the entire sample to predict the second score for each individual. Figure 4 shows the scatter plots of the individual data.

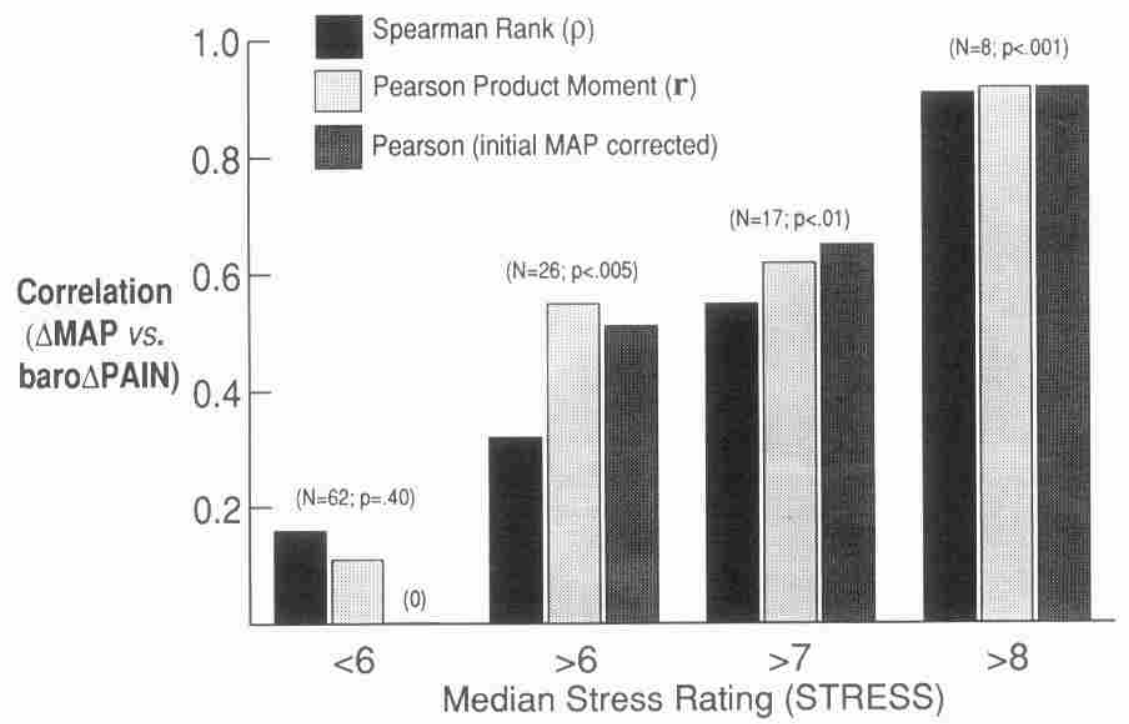

FIGURE 3 The baro $\triangle$ PAIN - $\triangle$ MAP correlations in subgroups of subjects reporting different median STRESS levels. The similarity between the Pearson Product Moment and Spearman Rank correlations indicates that the effects are not likely due to a few extreme cases, and comparing the raw and regression corrected difference scores (Cronbach \& Furedy, 1970) shows that although initial MAP probably contributed to the weak correlation in the low STRESS subjects, it was not involved in the much larger correlations in high STRESS subjects. (The baroreceptor reward mechanism is hypothesized to function only in subjects who experience substantial stress.) The $p$ value above each group of columns is the reliability for the raw score Pearson $r$. 


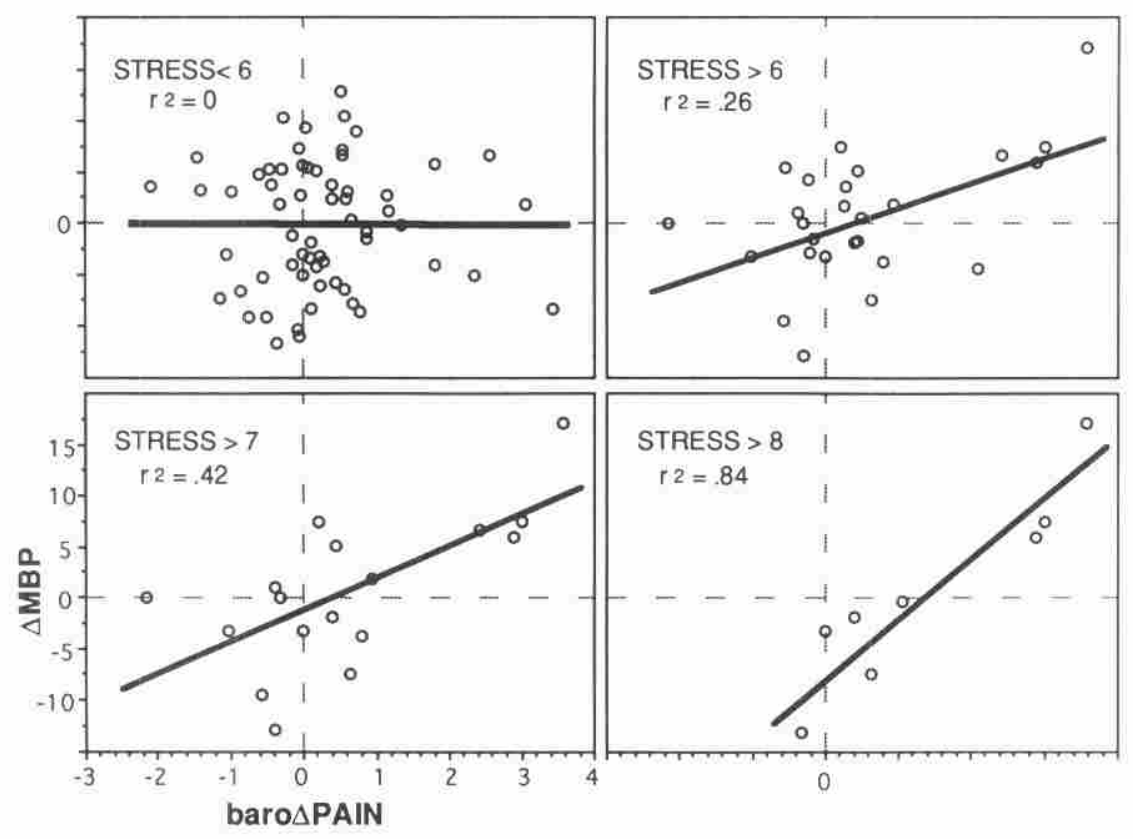

FIGURE 4 Scatter plots corresponding to the STRESS levels of the bar graphs. Each plot shows the change in mean BP during 20 months ( $\triangle \mathrm{MBP}$ ) versus the individual's baroreceptor pain inhibition effect (baro $\triangle$ PAIN).

\section{DISCUSSION}

In a previous report (Dworkin et al., 1994) we showed that in humans, carotid baroreceptor stimulation reduces both the pain from noxious stimulation and the amplitude of somatic reflexes. Our present result shows that, particularly for high stress individuals, the magnitude of the pain reduction effect predicts the increase in BP 20 months later. Together, these results indicate that the inhibitory corticofugal component of the baroreflex can potentially affect BP in two related but functionally and temporally distinct ways.

1. To the extent that it reduces perception of and reaction to aversive stimuli, barostimulation can immediately attenuate the direct effect of such stimuli on BP.

2. To the extent that, against a background of stress, it resembles and functions as a behavioral reward, repeated episodes of barostimulation, over time, can gradually strengthen learnable BP-elevating behaviors that have consistently preceded it. 
It is noteworthy that both of these properties are shared by most rewarding stimuli (Miller, 1959): For example, food immediately reduces the motivation of hunger and gradually rewards behaviors that directly preceded eating; water immediately reduces the motivation of thirst and gradually rewards behaviors that preceded drinking; and heroin promptly relieves the motivation of pain and anxiety but gradually rewards the behaviors of self-administration.

The presence of any strong motivation or drive increases the likelihood of a broad range of candidate behaviors, among which some would be far more efficient than others in reducing the motivation. When an effective behavior occurs, its motivation-reducing effect briefly lowers the immediate likelihood of all behaviors, including itself, while the associated reward effect simultaneously increases the future likelihood of only the effective behavior. These two effects work in tandem as a response selection mechanism to suppress ineffective behaviors and gradually strengthen learnable behaviors that efficiently reduce specific motivation (Dworkin, 1993). Applied to psychosomatic symptoms, such as essential hypertension, the response selection mechanism gives trial and error learning a means for symptom specificity that general reactivity models lack (Pickering \& Gerin, 1990). It explains, for example, why individuals with high STRESS, but low Baro $\triangle$ PAIN, probably would not learn to raise BP, but might instead learn some other stress-reducing symptom or coping behavior that would be more effective (Dworkin, 1988, 1991).

In hypertension, because of receptor adaptation and chronic resetting, baroreceptor activity is not elevated. An accepted implication of this is that the baroreceptors, as sensory elements in a conventional negative feedback loop, are not involved in maintaining high BP. However, the behavioral reinforcement, and feedback regulatory effects of baroreceptor activation are distinct neural mechanisms. Although they share the same receptors, they work quite differently. To maintain a tonic efferent regulatory output, such as vasoconstriction, a conventional negative feedback mechanism would require continuous, stimulus-dependent firing of the sensory receptor within the control loop, and because of resetting, for the baroreceptors, this presumably does not happen (see Chapter 7 of Dworkin, 1993, for a detailed analysis of the regulatory limitations imposed by receptor adaptation, and Chapter 8 for the possible role learning has in circumventing these). In contrast, delivery of behavioral reinforcement requires only periodic baroreceptor-produced central inhibition; thus, adaptation or resetting is not inconsistent with intermittent baroreceptor activation, rewarding, and strengthening through learning - tonic behaviors that sustain elevated BP. In fact, resetting would be expected to contribute positively to behavioral reward effects because, with sustained elevated BP, when the BP-baroreceptors firing curve shifts toward higher thresholds, the same amount of baroreceptor firing and stress or pain inhibition will necessitate behavior that produces higher BPs (see Dworkin, 1991, pp. 238-241). By analogy, the 
temporal course of narcotic tolerance resembles baroreceptor resetting; yet, heroin self-administration is readily learned and maintained against a background of growing tolerance to the drug, and, in fact, also similar to resetting, tolerance almost certainly pushes the addict toward larger, more frequent doses.

The hypothesis that baroreceptor CNS inhibition of pain and/or anxiety might reward antecedent BP-elevating behaviors through trial and error learning and thus contribute to hypertension was first proposed in 1977 (Miller \& Dworkin, 1977), and animal experiments showing quantitative behavioral effects of specific baroreceptor activation were reported in 1979 (Dworkin et al., 1979). This study was intended to further evaluate the hypothesis in normotensive human volunteers: As predicted, we found a correlation between baroreceptor pain inhibition and long-term BP changes, which strikingly increased in proportion to the amount of perceived life stress. We interpret this result to be evidence of a novel behavioral mechanism by which chronic exigencies from the environment could gradually raise a sensitive individual's basal BP and contribute to the development of essential hypertension.

\section{REFERENCES}

Benson, H., Herd, J. A., Morse, W. H., \& Kelleher, R. T. (1969). Behavioral induction of arterial hypertension and its reversal. American Journal of Physiology, 217, 30-34.

Cronbach, L. F., \& Furedy, L. (1970). How we should measure "change"-or should we? Psychological Bulletin, 74, 68-80.

Dworkin, B. R. (1988). Hypertension as a learned response: The baroreceptor reinforcement hypothesis. In T. Elbert, W. Langosch, A. Steptoe, \& D. Vaitl (Eds.), Behavioral medicine in cardiovascular disorders (pp. 17-47). Chichester: Wiley.

Dworkin, B. R. (1991). The baroreceptor reinforcement instrumental learning (BR-1L) model of essential hypertension: Biological data, quantitative mechanisms, and computer modeling. In A. Shapiro \& A. Baum (Eds.), Perspectives in behavioral medicine: Behavioral aspects of cardiovascular disease (pp. 213-245). Hillsdale, NJ: Lawrence Erlbaum Associates, Inc.

Dworkin, B. R. (1993). Learning and physiological regulation. Chicago: University of Chicago Press.

Dworkin, B. R., Elbert, T., Rau, H., Birbaumer, N., Pauli, P., Droste, C., \& Brunia, C. H. M. (1994). Central effects of baroreceptor activation in humans: I. Attenuation of skeletal reflexes and pain perception. In Proceedings of the National Academy of Sciences, USA, 91 . $6329-6333$.

Dworkin, B. R., Filewich, R. J., Miller, N. E., Craigmyle, N., \& Pickering, T. G. (1979). Baroreceptor activation reduces reactivity to noxious stimulation: Implications for hypertension. Science, 205, 1299-1301.

Eckberg, D. L., \& Sleight, P. (1992). Human baroreflexes in health and disease. Oxford, England: Clarendon.

Graham, D. T., Lundry, R. M., Benjamin, L. S., Kabler, J. D., Lewis, W. C., Kunish, N. O., \& Graham, F. K. (1962). Specific attitudes in initial interviews with patients that have different "psychosomatic" diseases. Psychosomatic Medicine, 24, 257-266.

Harris, A. H., Gilliam, W. J., Findley, J. D., \& Brady, J. V. (1973). Instrumental conditioning of large-magnitude, daily, 12-hour blood pressure elevations in the baboon. Science, 182, 175177. 
Miller, N. E. (1959). Liberalization of basic S-R concepts: Extensions to conflict behavior, motivation and social learning. In I. S. Koch (Ed.), Psychology: A study of a science (pp. 196-292). New York: McGraw-Hill.

Miller, N. E., Davis, J. D., \& Lulenski, G. C. (1968). Comparative studies of barbiturate self-administration. Journal of the Addictions, 3, 207-214.

Miller, N. E., \& Dworkin, B. R. (1977), Critical issues in therapeutic applications of biofeedback. In G. E. Schwartz \& J. Beatty (Eds.), Biofeedback: Theory and research (pp. 129-161). New York: Academic.

Neter, J., Wasserman, W., \& Kutner, M. H. (1985). Applied linear statistical models (2nd ed.). Homewood, IL: Irwin,

Obrist, P. A., Light, K. C., James, S. A., \& Strogatz, D. S. (1987). Cardiovascular responses to stress: I. Measures of myocardial response and relationship to high resting systolic pressure and parental hypertension. Psychophysiology, 24, 65-78.

Pickering, T. G. (1991). Ambulatory monitoring and blood pressure variability. London: Science Press.

Pickering, T. G., Brucker, B., Frankel, H. L., Mathias, C. J., Dworkin, B. R., \& Miller, N. E. (1977). Mechanisms of learned voluntary control of blood pressure in patients with generalized bodily paralysis. In J. Beatty \& H. Legewie (Eds.), Biofeedback and behavior (pp. 225-234). New York: Plenum.

Pickering, T. G., \& Gerin, W. (1990). Cardiovascular reactivity in the laboratory and the role of behavioral factors in hypertension: A critical review. Annals of Behavioral Medicine, 12, 3-16.

Plumlee, L. (1969). Operant conditioning of increases in blood pressure. Psychophysiology, 6. 283-290.

Sutherland, D. H. (1984). Gait disorders in childhood and adolescence. Baltimore: Williams \& Wilkins.

Talan, M. 1., \& Engel, B. T. (1986). Learned control of heart rate during dynamic exercise in non human primates. Journal of Applied Physiology, 61, 545-553.

Turkkan, J. S., \& Harris, A. H. (1981). Shaping blood pressure elevations: An examination of acquisition. Behaviour Analysis Letters, 1, 97-106. 